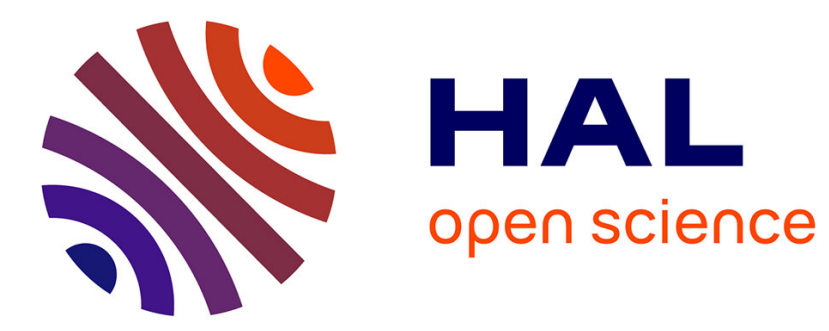

\title{
Sensor Deployment in Wireless Sensor Networks with Linear Topology using Virtual Node Concept
}

Rodrigue Domga Komguem, Razvan Stanica, Maurice Tchuenté, Fabrice Valois

\section{- To cite this version:}

Rodrigue Domga Komguem, Razvan Stanica, Maurice Tchuenté, Fabrice Valois. Sensor Deployment in Wireless Sensor Networks with Linear Topology using Virtual Node Concept. Wireless Networks, 2019, 25 (8), pp.4947-4962. 10.1007/s11276-019-02071-x . hal-02354755

\section{HAL Id: hal-02354755 \\ https://hal.inria.fr/hal-02354755}

Submitted on 7 Nov 2019

HAL is a multi-disciplinary open access archive for the deposit and dissemination of scientific research documents, whether they are published or not. The documents may come from teaching and research institutions in France or abroad, or from public or private research centers.
L'archive ouverte pluridisciplinaire HAL, est destinée au dépôt et à la diffusion de documents scientifiques de niveau recherche, publiés ou non, émanant des établissements d'enseignement et de recherche français ou étrangers, des laboratoires publics ou privés. 


\title{
Sensor Deployment in Wireless Sensor Networks with Linear Topology using Virtual Node concept
}

\author{
Rodrigue Domga Komguem*†, Razvan Stanica*, Maurice Tchuente ${ }^{\dagger}$, Fabrice Valois* \\ *INSA Lyon, CITI-Inria, F-69621, Villeurbanne, France \\ †Université de Yaoundé I, CETIC, LIRIMA, Faculté des Sciences, BP 812, Yaoundé, Cameroun
}

\begin{abstract}
Résumé-In a multi-hop wireless sensor network with a convergecast communication model, there is a high traffic accumulation in the neighborhood of the sink. This area constitutes the bottleneck of the network since the sensors deployed withing it rapidly exhaust their batteries. In this paper, we consider the problem of sensors deployment for lifetime maximization in a linear wireless sensor network. Existing approaches express the deployment recommendations in terms of distance between consecutive sensors. Solutions imposing such constraints on the deployment may be costly and difficult to manage. In this paper, we propose a new approach where the network is formed of virtual nodes, each associated to a certain geographical area. An analytical model of the network traffic per virtual node is proposed and a greedy algorithm to calculate the number of sensors that should form each virtual node is presented. Performance evaluation shows that the greedy deployment can improve the network lifetime by up to $40 \%$, when compared to the uniform deployment. Moreover, the proposed approach outperforms the related work when complemented by a scheduling algorithm which reduces the messages overhearing. It is also shown that the lifetime of the network can be significantly improved if the battery capacity of each sensor is dimensioned taking into account the traffic it generates or relays.
\end{abstract}

Keywords-Linear Wireless Sensor Network, Deployment, Energy Efficiency, Virtual Node, Greedy Algorithm, Lifetime.

\section{INTRODUCTION}

The topology and architecture of wireless sensor networks (WSN) generally depend on the target application and the geographical area where sensors are deployed. A linear WSN (LWSN) is a special case, where the physical topology of the network is a line [1]. The applications of LWSN are diverse, e.g. monitoring of large infrastructure such as bridges [2] and dams [3], road traffic observation [4], and border control [5]. Sensors typically operate on a small capacity battery and are thus limited in their active lifetime. It may be infeasible or expensive to change batteries in sensors once a wireless sensor network is deployed. It is therefore important to design energyefficient communication protocols and to optimize deployment strategies.

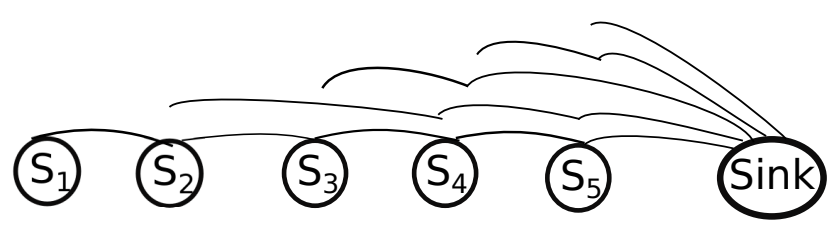

FIGURE 1: A LWSN of five sensors and one sink with convergecast traffic

In this paper, we are interested in sensors deployments. The goal of this paper is to present an energy-efficient and simple deployment strategy which can allow a network to operate for a long time. In a multi-hop LWSN with convergecast traffic (see Fig. 1), the amount of messages that a sensor has to forward significantly increases as the distance to the sink becomes smaller [6],[7]. Moreover, sensors are more exposed to the overhearing phenomenon : a sensor can receive a message addressed to another one. Thus, sensors closest to the sink, because of their high traffic load and their high overhearing probability, tend to exhaust their batteries early, causing network failure. This problem can be addressed through suitable deployment, by designing energy-efficient protocols or by considering non homogeneous energy distribution.

Many sensors deployment solutions are proposed in the literature [8]-[11]. In these solutions, message receptions are not always taken into account, although transmission and reception consume more or less the same energy. Moreover, deployment recommendations are usually expressed in terms of distances between consecutive sensors. For a LWSN with a large number of sensors, a solution with such an accuracy on the inter-node distances may be costly and difficult to implement.

In a LWSN, an event that occurs in an area can be detected by many sensors deployed in that area. A new deployment approach in which the LWSN is divided into virtual nodes and where consecutive virtual nodes should be wirelessly connected is proposed in this paper. An event happening within the area covered by a virtual node can be detected by any sensor, no matter its position in the virtual node. This paper also proposes a greedy algorithm for calculating the number of physical sensors to deploy for a virtual node, taking into account the traffic per virtual node. It is assumed that an average transmission power which guarantees connectivity between consecutive virtual nodes is used by sensors.

The concept of virtual node or virtual sensor is not new in the field of WSN. It is particularly used in the context of WSN programming [12]-[14]. In [13], every component represents a processing task applied to a stream of data originated from physical sensors and can be modeled as a virtual node. In [12], a virtual node corresponds either to a data stream received directly from sensors or to a data stream derived from other virtual sensors. In this paper, the concept of virtual node is used in the context of sensors deployment to reduce the complexity of the deployment.

The solution proposed here is different from the existing ones on at least two points. First, there is no need to keep a precise distance between sensors. Indeed, after dividing the network into virtual nodes (the number of virtual nodes 
is smaller than the number of physical nodes), the position of sensors within a virtual node is not predefined. Second, contrarily to our solution, which considers also message receptions, the related work only account for message transmissions. This is not realistic because it is well known that most radio modules consume almost the same energy for receptions and transmissions [15]. It is important to note that message receptions mentioned here include receptions due to the overhearing phenomenon.

Results presented in this paper show that, by properly selecting the number of sensors, a simple greedy deployment can improve the network lifetime by up to $40 \%$, when compared to the uniform deployment. A framework which allows to compare the virtual node-based approach proposed in this paper to the distance-based one defined in [8] is also designed. Results show that our approach outperforms that of [8] when a scheduling algorithm which reduces the messages overhearing is used.

A solution for heterogeneous networks where the battery capacities of sensors are not uniform is also proposed. More precisely, it is assumed that the battery capacity of a sensor is proportional to its traffic load. Results show that this solution significantly improves the network lifetime compared to the homogeneous case.

The remainder of this paper is organized as follows. Section II presents related work on sensor deployment. The virtual node approach is formalized in Section III and Section IV describes a greedy algorithm for sensor deployment. Section $\mathrm{V}$ is devoted to performance analysis. In Section VI, the comparison to the related work is done, and Section VII considers the case of heterogeneous networks. Finally, Section VIII concludes this paper.

\section{RELATED WORK}

In a multi-hop WSN with a convergecast communication model, the sensors close to the sink have a high transmission activity. Thus, they constitute the bottleneck of the network, since sensors are constrained in terms of battery (limited capacity), memory (limited buffer size) and communication capabilities (high contention). Many energy-efficient solutions have been proposed at all layers of the communication stack, ranging from hardware design, MAC and routing protocols, and data aggregation [16]. In this paper, we are interested in a sensor deployment strategy which could prolong the network lifetime.

Sensor deployment strategies can be designed with different objectives in mind. For example, the objective of the deployment can be to guarantee a balanced energy consumption of sensors in the network. To address this problem, one can deploy more sensors close to the sink and make them transmit at lower power levels [8]-[10]. In this way, sensors close to the sink transmit more messages, but consume less energy per message. When all sensors use the same transmission power, in network areas with high sensors density, a message can be forwarded by a significant number of sensors. Load-balancing can then be achieved by adopting duty-cycle scheduling [17].

The problem of sensors deployment is widely addressed in the literature [18]-[23]. In [23], authors propose a hybrid differential evolution and simulated annealing (DESA) algorithm for clustering and choice of cluster heads. They prolong the network lifetime by appropriately selecting the cluster head and affecting sensors to clusters. [18] addresses the optimal deployment problem defined as the determination of the minimum possible number of sensors aiming to achieve the targeted partial connected coverage, the lowest financial cost and the the highest lifetime. In [22], authors propose a novel node deployment strategy based on Quasi-random method of low-discrepancy sequences to increase the lifetime and the coverage of the network. While most of the previous work consider 2-D or 3-D topologies, this paper focues on LWSN, i.e. a WSN with 1-D topology. Rather than consider a hierarchical network (like in clustered networks), we are interested by flat WSN in which all the messages generated by sensors are forwarded in a multi-hop manner toward the sink deployed at an network edge.

In [7], authors propose an analytic description of the traffic over a linear, randomly deployed WSN. They evaluate the effect of the number of sensors and their distribution on network traffic. They propose a non uniform deployment obtained through an increased network density closer to the sink. Their results show that, given a number $N$ of sensors, such a deployment can significantly reduce the maximum traffic load per node compared to a uniform deployment, and hence improve the network lifetime. However, the authors do not propose a deployment strategy or some concrete recommendations for sensors deployment.

In [11], the problem of sensor deployment is addressed when all sensors have the same transmission power level. Given the required lifetime of a sensor network, the energy constraint of sensors, and the area to be covered, authors study the problem of finding the minimum number of sensors needed to build such a network and the corresponding deployment scheme. In [8], the authors assume that a sensor can select the transmission power among a set of power levels. Given the energy constraint of sensors, the problem of sensors deployment is addressed with the goal of maximizing the covered area and the network lifetime. They formalize the deployment problem as a Mixed-Integer Linear Programming (MILP) problem. Their results show that, by properly selecting the number of sensors, the distance between them, and the corresponding transmission power, the WSN lifetime can be improved by up to $30 \%$.

Despite their performance in terms of network lifetime, a drawback of the solutions proposed by [8], [11] is the complexity of their implementation, particularly in large LWSN. Indeed, the deployment recommendations are expressed in terms of distances between consecutive sensors in the network. This is not practical if the deployment must be done by a human for a large network. In this paper, we focus on a solution that is simple to implement, i.e. it requires reduced deployment effort, and can at the same time improve significantly the network lifetime compared to a uniform deployment. Another important contribution with respect to the state of the art is that, because of the broadcast nature of the wireless communication channel, the proposed solution takes into account messages received due to the overhearing phenomenon. 
TABLE I: Model Notations

\begin{tabular}{|c|l|}
\hline Parameter & Description \\
\hline$N$ & Number of sensors \\
\hline$\lambda$ & Number of virtual nodes \\
\hline$\Delta$ & $\begin{array}{l}\text { Avg. number of messages generated per virtual node per } \\
\text { time slot }\end{array}$ \\
\hline$p$ & Time slot duration \\
\hline$\alpha$ & $\begin{array}{l}\text { Probability for the virtual node } B_{i} \text { to receive messages from } \\
\text { virtual node } B_{i-2} \text { or } B_{i+2}\end{array}$ \\
\hline$E_{\text {sensor }}$ & $\begin{array}{l}\text { Probability for a sensor to receive messages destined to } \\
\text { another sensor }\end{array}$ \\
\hline$P$ & The sensor battery capacity \\
\hline$E_{i}$ & $\begin{array}{l}\text { Energy consumed per sensor per time slot in the virtual node } \\
B_{i}\end{array}$ \\
\hline$\tau$ & The time required to transmit one packet \\
\hline$T_{i}$ & $\begin{array}{l}\text { The total number of transmissions from a virtual node } B_{i}: \\
\text { generated and relayed messages }\end{array}$ \\
\hline$n_{i}$ & Number of sensors in the virtual node $B_{i}$ \\
\hline $\bar{R}_{i}^{n S}$ & $\begin{array}{l}\text { Avg. number of receptions per sensor in virtual node } B_{i} \\
\text { when the radio of nodes is always on }\end{array}$ \\
\hline $\bar{R}_{i}^{\text {os }}$ & $\begin{array}{l}\text { Avg. number of receptions per sensor in virtual node } B_{i} \\
\text { when there is no overhearing }\end{array}$ \\
\hline $\bar{R}_{i}^{p s}$ & $\begin{array}{l}\text { Avg. number of receptions per sensor in virtual node } B_{i} \\
\text { when the overhearing is partial }\end{array}$ \\
\hline $\bar{O}_{i}$ & $\begin{array}{l}\text { Avg. number of operations per sensor per time slot in the } \\
\text { virtual node } B_{i}\end{array}$ \\
\hline
\end{tabular}

\section{PROBLEM DESCRIPTION}

Given a set of homogeneous sensors in terms of battery capacity, our goal is to design a simple, energy-efficient and realistic sensor deployment that maximizes the network lifetime. In Section VII, we investigate the case where sensors are heterogeneous.

In many WSNs applications, the same event can be detected by many sensors if the network is dense. For instance, if a WSN is deployed to monitor the vehicular traffic on a highway or on lanes of an intersection, a vehicle might be detected at the same time by different sensors. This depends on the average vehicle length and the distance between two consecutive nodes. Another example is the monitoring of the status of an infrastructure like a bridge. In such an application, a damage on an area of the bridge could be detected by many sensors. Thus, rather than considering a flat network like in related work [8], [11], we consider a large network divided into virtual nodes wirelessly connected. A virtual node represents the set of sensors deployed in a given area which can measure the same physical phenomenon. In the related work, the deployment recommendations is expressed in terms of distance between consecutive sensors. In our approach, once the virtual nodes are defined, the position of sensors in a virtual nodes are not predefined. In the next section, our hypothesis are further detailed. Tab. I presents the list of important notations used in our model.

\section{A. Assumptions}

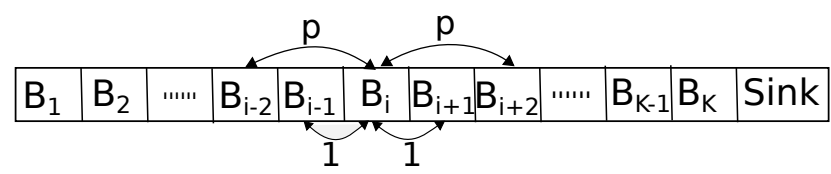

FiguRE 2: Network Model

We consider a set of $N$ sensors $\left\{S_{1}, \cdots, S_{N}\right\}$ deployed in a $1 D$ space to monitor a phenomenon. We assume that the area to be monitored is covered by $K$ virtual nodes $\left\{B_{1}, \cdots, B_{K}\right\}$, as presented in Fig. 2. We also assume that the sink is a single node which corresponds to the virtual node $B_{K+1}$. In our work, we assume that consecutive virtual nodes are connected, in order to guarantee that a message generated in a virtual node can be received by the sink. However, we note that this assumption does not imply that physical nodes use homogeneous power levels, since the physical distance between nodes is not necessarily uniform, and several physical nodes can form a virtual node.

An event happening in an area can be detected by many sensors. We consider that for each event detected by a virtual node, only one message will be generated, as a result of a cooperation algorithm executed by sensors of the same virtual node. This cooperation algorithm also equally distributes to the nodes in $B_{i}$ the traffic coming from $B_{j}, j<i$. We also assume that sensors can run a ranking algorithm [24] to determine the relative position of each sensor to the sink. Such an algorithm allows sensors to compute the virtual node index in which they are deployed.

\section{B. Connectivity model and traffic pattern}

It is assumed a multi-hop LWSN with a convergecast communication model, i.e data from all sensors are forwarded toward the sink located at position $K+1$. Unlike a distributed peer-to-peer wireless network, the traffic load is highly asymmetric, i.e. sensors closer to the sink have a heavier relay load. A wireless connectivity is assumed between consecutive virtual nodes $B_{i}$ and $B_{i+1}$. A virtual node $B_{i}$ can communicate with $B_{i+2}$ or $B_{i-2}$ with probability $p$ (see Fig. 2). This models the an-isotropic property of the signal. We consider a shortest path routing protocol [26], i.e. a packet transmitted by $B_{i}$ and received by $B_{i+1}$ and $B_{i+2}$ is forwarded by $B_{i+2}$. The average number of messages generated from a virtual node per time unit of duration $\Delta$ is denoted by $\lambda$. Typically, $\lambda$ is application dependent and represents the average number of events detected by a virtual node during a time slot. $T_{i}$ denotes the total traffic load (messages generated or relayed) of virtual node $B_{i}$ per time unit. This traffic $\left(T_{i}\right)$ comes from three different sources :

- From $B_{i}: \lambda$ messages generated

- From $B_{i-1}:(1-p) \cdot T_{i-1}$ messages relayed

- From $B_{i-2}: p \cdot T_{i-2}$ messages relayed.

Thus, the traffic load of virtual node $B_{i}$ is given by Eq. 1 .

$$
T_{i}=\left\{\begin{array}{l}
\lambda, \text { if } i=1 \\
(2-p) \cdot \lambda, \text { if } i=2 \\
p \cdot T_{i-2}+(1-p) \cdot T_{i-1}+\lambda, \text { if } 2<i \leq K
\end{array}\right.
$$

Eq. 1 is a recurrence equation whose solution is given by Eq. 2 .

$$
T_{i}=\beta+\gamma \cdot(-p)^{i}+\frac{\lambda \cdot i}{p+1}, \forall i, 1 \leq i \leq K
$$

In Eq. 2, $\beta$ and $\gamma$ are constants calculated from $T_{1}$ and $T_{2}$ (see Eq. 1). Thus, $\beta=\frac{p \cdot \lambda}{(p+1)^{2}}$ and $\gamma=-\frac{p \cdot \lambda}{(p+1)^{2}}$. The traffic load in the virtual node $B_{i, 1 \leq i \leq K}$ is thus given by Eq. 3 .

$$
T_{i}=\frac{\lambda}{p+1} \cdot\left[\frac{p}{p+1}+\frac{(-p)^{i+1}}{p+1}+i\right], \forall i, 1 \leq i \leq K
$$

Thanks to the scheduling algorithm used, it is assumed a uniform distribution of traffic between sensors in a given 
virtual node. The average number of transmissions per sensor in the virtual node $B_{i}$, which contains $n_{i}$ sensors, is :

$$
\bar{T}_{i}=\frac{T_{i}}{n_{i}}, \forall i, 1 \leq i \leq K
$$

\section{Number of operations per sensor}

In a WSN, a sensor consumes energy during data sensing and processing, and through its radio for data transmission and reception. It is well known that the radio generally consumes most of the energy of the sensor [25]. In this paper, we denote as energy-consuming operations only the transmission and the reception of a packet. Thus, the number of operations executed by a sensor is the total number of messages sent and received by that sensor. The total number of operations per virtual node is equal to the number of operations of all the sensors which form the virtual node. But, since our goal is to maximize the sensor lifetime, in this section, we focus on the number of operations per sensor. We have already seen that the average number of transmissions per sensor deployed in a virtual node is given by Eq. 4 .

A sensor may receive a message destined to another sensor : it is the overhearing phenomenon [27]. Therefore, the number of receptions observed by a sensor depends on the scheduling algorithm used. We assume in this paper that the goal of the scheduling algorithm is to determine the time at which a sensor must switch its radio on or off. Thus, a perfect scheduling is when a sensor switches its radio on only when it has a message to send or to receive. In order to model the number of receptions per sensor per time period, different cases are considered in the following :

- No scheduling, where sensors keep their radio always on and ready to transmit/receive

- Optimal Scheduling, where the overhearing is eliminated by a perfect scheduling.

- Practical Scheduling, where the overhearing is partially, but not totally, eliminated.

1) Case 1 : When the radio is always on: It is assumed here that each sensor maintains its radio on all the time. In such a situation, a sensor will receive all transmissions in its communication range. This includes transmissions from neighboring virtual nodes and from the virtual node to which the sensor belongs. For a virtual node $B_{i}, 1 \leq i \leq K$, there are two sources of messages :

- Receptions from neighboring virtual nodes : this concerns transmissions from virtual nodes $i-2, i-$ $1, i+1$ and $i+2$. This quantity is equal to $p \cdot T_{i-2}+$ $T_{i-1}+T_{i+1}+p \cdot T_{i+2}$, since we assume that a sensor in a virtual node $i$ can always receive transmissions from virtual nodes $i-1$ and $i+1$, and it can communicate with virtual nodes $i-2$ and $i+2$ with a probability $p$. We define $T_{i}=0$ for $i<1$ and $i>K$.

- Messages transmitted by other sensors in the same virtual node $: T_{i}-\frac{T_{i}}{n_{i}}$. Indeed, for a traffic load $T_{i}$ in virtual node $B_{i}$, the average number of transmissions for a given sensor $s$ is given by Eq. 4. Then, the remaining messages $\left(T_{i}-\frac{T_{i}}{n_{i}}\right)$ will be transmitted by other sensors. Sensor $s$ will also receive these messages, since we assume each sensor continuously maintains its radio on.
The total number of receptions is then given by Eq. 5 .

$$
\bar{R}_{i}^{n s}=p \cdot T_{i-2}+T_{i-1}+T_{i+1}+p \cdot T_{i+2}+T_{i}-\frac{T_{i}}{n_{i}}
$$

Under the assumption of the radio always on, the average number of operations per sensor per time unit in a virtual node $B_{i}$ is the sum of transmissions (Eq. 4) and receptions (Eq. 5) per sensor and is expressed by Eq. 6 .

$$
\begin{aligned}
\bar{O}_{i} & =\bar{T}_{i}+\bar{R}_{i}^{n s} \\
& =\frac{T_{i}}{n_{i}}+p \cdot T_{i-2}+T_{i-1}+T_{i+1}+p \cdot T_{i+2}+T_{i}-\frac{T_{i}}{n_{i}} \\
& =p \cdot T_{i-2}+T_{i-1}+T_{i+1}+p \cdot T_{i+2}+T_{i}
\end{aligned}
$$

From Eq. 6, an interesting property appears : the average number of operations per sensor per time unit is independent of the number of sensors deployed in the virtual node. Thus, a simple solution is to consider the same number of sensors per virtual node ; this corresponds to a uniform deployment. Under the assumption of the radio always on, the optimal solution is to deploy one sensor in each virtual node, since it is useless to deploy more sensors in a virtual node.

2) Case 2 : Optimal scheduling: This section assumes an optimal scheduling algorithm where a sensor is able to switch its radio on only when there is a message to forward or only when it has a new message to transmit. If such a scheduling algorithm is used, there will be no overhearing and the messages received by a sensor will be only those it needs to relay for the previous virtual nodes. The traffic relayed by sensors in the virtual node $B_{i}$ is $T_{i}-\lambda$. Since we assume a uniform distribution of this traffic among sensors in the virtual node $B_{i}$, the average number of receptions per sensor is given by Eq. 7 .

$$
\bar{R}_{i}^{o s}=\frac{T_{i}-\lambda}{n_{i}}
$$

Thus, the average number of operations per sensor per time unit is the sum of transmissions (Eq. 4) and receptions (Eq. 7) and is given by Eq. 8

$$
\bar{O}_{i}=\bar{T}_{i}+\bar{R}_{i}^{o s}=\frac{T_{i}}{n_{i}}+\frac{T_{i}-\lambda}{n_{i}}=\frac{2 \cdot T_{i}-\lambda}{n_{i}}
$$

From Eq. 8, we observe that, when the number of sensors $n_{i}$ increases, $\bar{O}_{i}$ decreases. Thus by increasing the number of sensors deployed in a virtual node, the network functioning time increases too.

3) Case 3 : Practical scheduling: In a wireless network, because of multiple constraints (environment, hardware, communication technology, etc), scheduling algorithms ([27]-[29]) cannot be perfect. A sensor may receive a message addressed to another one : this is the overhearing phenomenon. Thus, if we consider a particular sensor $s$ deployed in the virtual node $B_{i}$, in addition to the messages transmitted by previous virtual nodes and relayed by $s, s$ may receive other transmissions in its communication range. We model this effect by a parameter $\alpha$. This parameter represents the ratio of messages transmitted in the communication area of a sensor $s$, not addressed to $s$, but received by $s$. In the following, $\alpha$ will be called the overhearing ratio. To calculate the total number of messages 
that $s$ can receive due to the overhearing phenomenon, the following components must be considered :

- Messages transmitted (generated or relayed) by sensors in the virtual node $B_{i-1}$, but relayed by sensors in the virtual node $B_{i+1}$

$$
\bar{R}_{i}^{r s n r}=p \cdot T_{i-1}
$$

- Messages transmitted (generated or relayed) by previous virtual nodes, relayed by sensors in the virtual node $B_{i}$, but a different node from $s$

$$
\bar{R}_{i}^{r s r}=\left(T_{i}-\lambda\right)-\frac{T_{i}-\lambda}{n_{i}}=\frac{\left(T_{i}-\lambda\right) \cdot\left(n_{i}-1\right)}{n_{i}}
$$

- Messages transmitted by other sensors in the virtual node $B_{i}$ :

$$
\bar{R}_{i}^{r s c}=T_{i}-\frac{T_{i}}{n_{i}}=T_{i} \cdot \frac{n_{i}-1}{n_{i}}
$$

- Messages transmitted by sensors in virtual nodes $B_{i+1}$ and $B_{i+2}$ :

$$
\bar{R}_{i}^{r s f}=T_{i+1}+p \cdot T_{i+2}
$$

Thus, the number of messages received by sensor $s$ due to overhearing is defined by Eq. 13

$$
\begin{aligned}
\bar{R}_{i}^{o} & =\alpha \cdot\left(\bar{R}_{i}^{r s r}+\bar{R}_{i}^{r s n r}+\bar{R}_{i}^{r s f}+\bar{R}_{i}^{r s c}\right) \\
& =\alpha \cdot\left[\frac{\left(T_{i}-\lambda\right) \cdot\left(n_{i}-1\right)}{n_{i}}+p \cdot T_{i-1}\right. \\
& \left.+T_{i+1}+p \cdot T_{i+2}+T_{i} \cdot \frac{n_{i}-1}{n_{i}}\right] \\
& =\alpha \cdot\left[\frac{\left(2 \cdot T_{i}-\lambda\right) \cdot\left(n_{i}-1\right)}{n_{i}}+p \cdot T_{i-1}\right. \\
& \left.+T_{i+1}+p \cdot T_{i+2}\right]
\end{aligned}
$$

To obtain the total number of receptions of $s$, we also consider the messages transmitted from previous virtual nodes and relayed by $s$ (see Eq. 7). Eq. 14 gives the average number of receptions per sensor deployed in a virtual node $B_{i}$.

$$
\begin{aligned}
\bar{R}_{i}^{p s} & =\bar{R}_{i}^{o s}+\bar{R}_{i}^{o}=\frac{T_{i}-\lambda}{n_{i}}+\alpha \cdot\left[\frac{\left(2 \cdot T_{i}-\lambda\right) \cdot\left(n_{i}-1\right)}{n_{i}}\right. \\
& \left.+p \cdot T_{i-1}+T_{i+1}+p \cdot T_{i+2}\right]
\end{aligned}
$$

The average number of operations per sensor per time unit is then given by the Eq. 15 .

$$
\begin{aligned}
\bar{O}_{i} & =\bar{T}_{i}+\bar{R}_{i}^{p s} \\
& =\frac{T_{i}}{n_{i}}+\frac{T_{i}-\lambda}{n_{i}}+\alpha \cdot\left[\frac{\left(2 \cdot T_{i}-\lambda\right) \cdot\left(n_{i}-1\right)}{n_{i}}\right. \\
& \left.+p \cdot T_{i-1}+T_{i+1}+p \cdot T_{i+2}\right] \\
& =\frac{\left(2 \cdot T_{i}-\lambda\right)}{n_{i}}+\alpha \cdot\left[\frac{\left(2 \cdot T_{i}-\lambda\right) \cdot\left(n_{i}-1\right)}{n_{i}}\right. \\
& \left.+p \cdot T_{i-1}+T_{i+1}+p \cdot T_{i+2}\right]
\end{aligned}
$$

Note that, the optimal scheduling corresponds to the case $\alpha=0$, whereas the radio always on corresponds to the case $\alpha=1$. It is also important to note that $\bar{O}_{i}$ decreases when $n_{i}$ increases. But when $n_{i}$ is very large : $\bar{O}_{i} \approx \bar{O}_{i}^{*}=\alpha \cdot\left(2 \cdot T_{i}-\right.$ $\left.\lambda+p \cdot T_{i-1}+T_{i+1}+p \cdot T_{i+2}\right)$. Thus, if $n_{i}=n_{i}^{*}$ then $\bar{O}_{i} \approx \bar{O}_{i}^{*}$, it is useless to deploy more than $n_{i}^{*}$ sensors in the virtual node $B_{i}$, since this will not improve the lifetime of sensors in $B_{i}$.

\section{Problem formulation}

Given $N$ sensors that form $K$ virtual nodes, the goal of this paper is to find the number $n_{i}$ of sensors in virtual node $B_{i}, 1 \leq i \leq K$, such that $\sum_{i=1}^{K} n_{i}=N$, and which minimizes the maximum number of operations executed by a sensor. Formally, the problem can be formulated as :

$$
\text { minimize } \max _{1 \leq i \leq K} \bar{O}_{i}
$$

subject to the following constraints :

$$
\begin{gathered}
\sum_{i=1}^{K} n_{i}=N \\
n_{i} \geq 1, i=1, \cdots, N
\end{gathered}
$$

Eq. 16-18 define a mixed-integer nonlinear programming problem. In the following section, given the number $N$ of sensors, we propose a greedy algorithm to calculate, for each virtual node $B_{i}$, the value of $n_{i}$.

\section{DEPLOYMENT ALGORITHM}

This work assumes that a sensor is declared dead when it exhausts its battery. This paper assumes a uniform load distribution within a virtual node. This means that all sensors in the same virtual node will die approximately at the same time. Thus, we define the network lifetime as the time until all sensors in a virtual node die (or, equivalently, until the first sensor dies). The network lifetime is maximized by reducing as much as possible the traffic load per sensor. The problem addressed in this paper can be formulated as follows : given $N$ sensors forming $K$ virtual nodes, how many sensors should be deployed in each virtual node in order to maximize the network lifetime? Hereafter, the average number of operations per sensor per time unit in the virtual node $B_{i}$ is denoted $\overline{O_{i}}$ (see Eq. 15). We propose in Algorithm 1 a greedy approach to compute the number of sensors to deploy in each virtual node.

Recall that, in each virtual node, we will have at least one sensor, for connectivity and sensing purposes. That is why the number $N$ of sensors is greater than or equal to the number $K$ of virtual nodes. Initially, one sensor is assigned to each virtual node (Line 4) and the remaining sensors are iteratively deployed. At each iteration, one sensor is added to the virtual node which has the highest $\bar{O}_{i}$. If more than one virtual node have the maximum number of operations per sensor per time unit, the one with the highest index is selected (Lines 10$11)$ : it is the one which is closest to the sink. Indeed, the traffic is highest as the virtual node is closer to the sink. The consequence of adding a sensor to a virtual node $B_{i}$ is the reduction of the average number of operations per sensor per time unit in that virtual node. Since our objective is to 


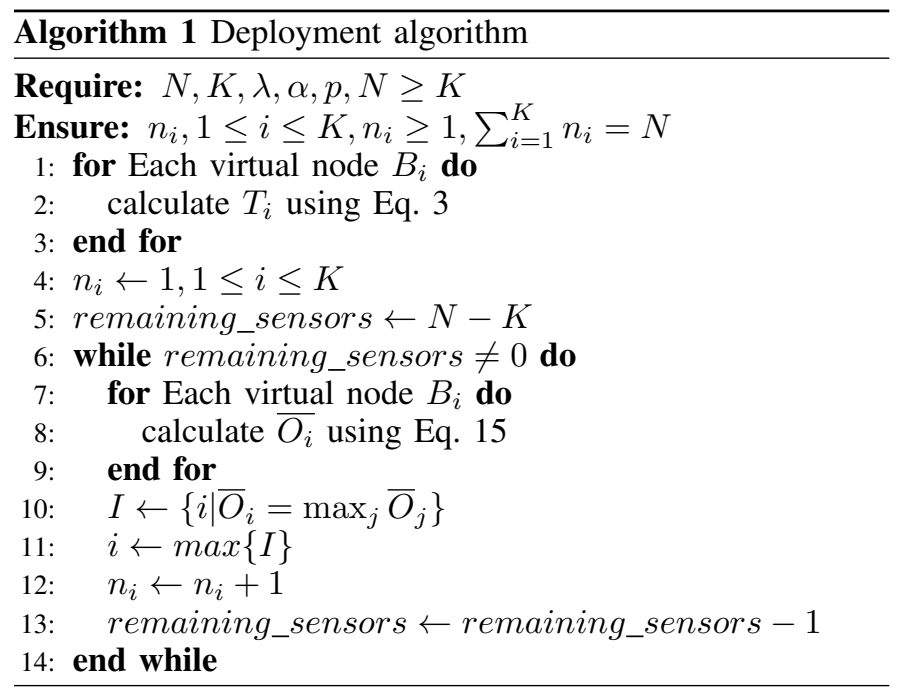

propose a deployment which minimizes the maximum number of operations per sensor, the problem can be decomposed in a series of sub-problems, which are optimally solved iteratively by the greedy Algorithm 1, resulting in an optimal general solution.

At the beginning of this algorithm, the traffic of each virtual nodes is calculated. We assume that, for a virtual node $B_{i}$, $T_{i}$ is calculated in $\mathrm{O}(K)$. Thus, calculating this value for all virtual nodes is done in $\mathrm{O}\left(K^{2}\right)$. In the iterative part of the algorithm, at each iteration, the average number of operations per sensor per virtual node is calculated. This processing can be done in $\mathrm{O}(K)$. Since the goal of the iterative part of the deployment algorithm is to find the virtual node in which each of the $N-K$ (since initially one sensor is deployed per virtual node) virtual nodes will be deployed, the iterative part of our algorithm can be executed in $\mathrm{O}(N \cdot K)$. Thus, the result of the deployment is computed in $\mathrm{O}\left(K^{2}+N \cdot K\right)$. Even for a large network with a high number of sensors, it is possible to rapidly obtain the number of sensors which forms each virtual node, since this algorithm can be executed on a computer with good performance, and not on the nodes themselves.

\section{ANALYTICAL RESUlTS}

This section presents analytical results concerning the virtual node-based approach proposed in this paper. These results concern the greedy deployment described by Algorithm 1 and a simple uniform deployment. In the later one, the same number of sensors are deployed in all virtual nodes. Firstly (see Section V-B), we compare the two deployments in terms of sensors distribution for different parameters. Secondly (see Section V-C), the two deployments are compared in terms of network lifetime. The next section describes the evaluation parameters.

\section{A. Evaluation parameters}

Without loss of generality, it is assumed that the average output power is $P=61.9 \mathrm{~mW}$, which is the maximum transmission power of the radio used by Tmote Sky [8]. It is also assumed that each sensor has a battery capacity of $E_{\text {node }}=5 \mathrm{Ah}$. If we assume a packet size of 128 Bytes and a data rate of $250 \mathrm{kbps}$, the time $\tau$ to transmit one packet is equal to $4.096 \mathrm{~ms}$. Thus, $E_{i}$, the average energy consumed by a sensor in the virtual node $B_{i}$ per time unit is defined by Eq. 19.

$$
E_{i}=\bar{O}_{i} \cdot \tau \cdot P
$$

Tab. II summarizes the values of parameters we use to obtain numerical results.

TABLE II: Evaluation parameters

\begin{tabular}{|c|l|c|}
\hline Parameter & Description & Value \\
\hline$E_{\text {node }}$ & The sensor battery capacity & $5 \mathrm{Ah}$ \\
\hline$P$ & Average transmission power & $61.9 \mathrm{~mW}$ \\
\hline$\tau$ & Time to transmit one message & $4.096 \mathrm{~ms}$ \\
\hline$\Delta$ & Time slot duration & $5 \mathrm{~s}$ \\
\hline$\lambda$ & $\begin{array}{l}\text { Avg. number of messages generated per } \\
\text { virtual node per time slot }\end{array}$ & 1 message \\
\hline
\end{tabular}

\section{B. Spatial sensors distribution}

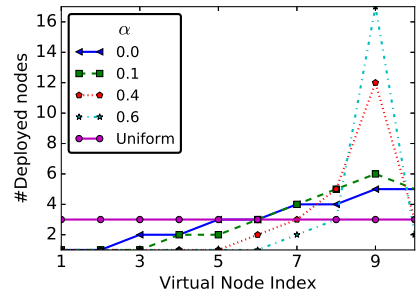

(a) $p=0.0$

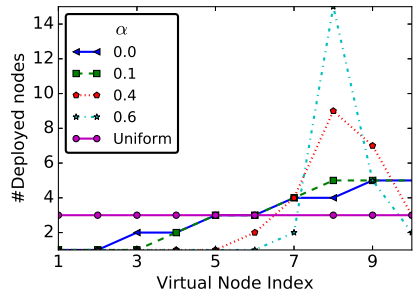

(b) $p=0.5$
FIGURE 3: Number of nodes deployed in each virtual node : $N=30, K=10$.

Fig. 3 presents the sensors distribution obtained by a uniform deployment and the greedy Algorithm 1. It gives the number of sensors forming each virtual node as a function of the virtual node position and the overhearing ratio $(\alpha)$ when we consider $N=30$ sensors to deploy in a network of $K=10$ virtual nodes. Note that, when the number of virtual nodes is 10 , the sink is deployed alone in a particular virtual node (not represented in Fig. 3) at the position $K+1$. Fig. 3 a and $3 \mathrm{~b}$ are for $p=0$ and $p=0.5$ respectively ( $p$ being the probability of a sensor in the virtual node $B_{i}$ to receive messages from $B_{i-2}$ or $B_{i+2}$ ).

With the greedy deployment proposed by Algorithm 1, we observe that there is always a virtual node $B_{i *}$ which receives the highest number of sensors. The position of this virtual node depends on $p$ and is independent of $\alpha$. When $p=0.0, B_{9}$ is the bottleneck of the network (highest traffic) and receives the maximum number of sensors (Figure 3a). Indeed, unlike sensors in $B_{10}$ which receive messages only from the left side, sensors in $B_{9}$ receive messages from both the left and the right side. When $p=0.5$ (Fig. $3 \mathrm{~b}$ ), the virtual node $B_{8}$, which receives messages from "two-hop" virtual nodes, becomes the bottleneck of the network and is assigned the maximum number of sensors. For other virtual nodes which are farther from the sink than $B_{i *}$, the number of sensors deployed in a virtual node increases linearly as a function of the virtual node index.

When only transmissions are considered, like in most of the related work, the last virtual node of the network will always receive the maximum number of sensors. Since we 
consider transmissions and receptions of messages, our results show a different trend. In Fig. 3, we also observe that, when the overhearing ratio $(\alpha)$ increases, the number of sensors deployed in the virtual node $B_{i *}$ increases too. Indeed, the traffic is highest in this virtual node and a high value of $\alpha$ results in a high number of receptions.

In summary, when the overhearing ratio is not taken into account $(\alpha=0)$, the number of sensors per virtual node is almost a linear increasing function of the virtual node index. When the overhearing is taken into account $(\alpha>0)$, the number of sensors deployed per virtual node increases until a virtual node $B_{i *}$ which receives the highest number of sensors, and then drastically decreases. The position of $B_{i *}$ depends on $p$. If $p=0, B_{i *}=B_{K-1}$ and when $p>0, B_{i *}=B_{K-2}$.

\section{Lifetime : Comparison with uniform deployment}

In a uniform deployment, the same number of sensors is deployed in all virtual nodes. We consider as our baseline the network lifetime $L T^{\text {(uniform })}$ obtained with such a deployment. Since we assume that the traffic is balanced between sensors in a virtual node, the lifetime of a virtual node is equal to the average lifetime of sensors deployed in that virtual node. We denote by $E_{\max }=\max \left\{E_{i} \mid i=1, \cdots, K\right\}$ the maximum energy consumed by a sensor per time unit in the network. Recall that $E_{i}$ is the energy consumption rate per sensor in the virtual node $B_{i}$ (see Eq. 19). If $E_{\text {node }}$ is the initial energy of a sensor, for a given deployment, the lifetime of the network will be equal to $\frac{E_{\text {node }}}{E_{\max }} \cdot \Delta$.

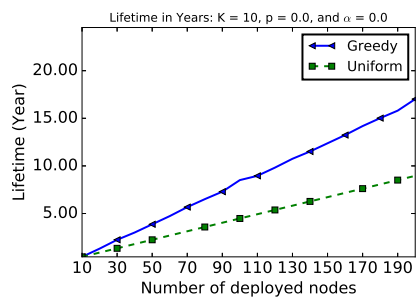

(a) $p=0, \alpha=0$

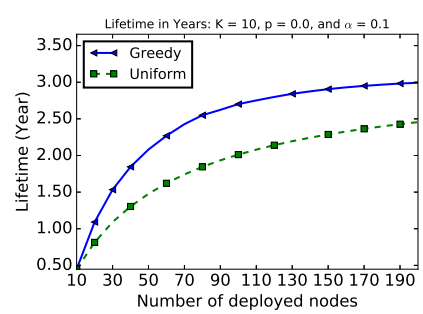

(c) $p=0, \alpha=0.1$

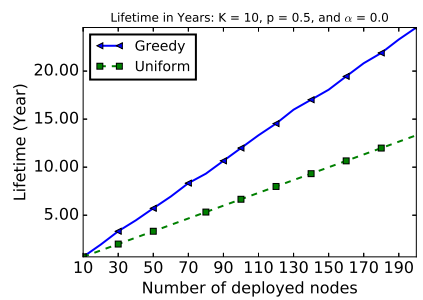

(b) $p=0.5, \alpha=0$

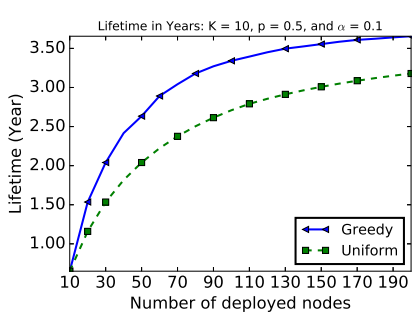

(d) $p=0.5, \alpha=0.1$
FIGURE 4: Impact of the number of nodes on the lifetime for different values of $\alpha$ and $p$

1) The lifetime of the greedy and uniform deployment:

Fig. 4 presents the lifetime of the greedy and the uniform deployment when $p=0$ and $\alpha=0$ (Fig. 4a), $p=0.5$ and $\alpha=0$ (Fig. 4b), $p=0$ and $\alpha=0.1$ (Fig. 4c) and $p=0.5$ and $\alpha=0.1$ (Fig. $4 \mathrm{~d}$ ). The results presented in this figure show that the greedy deployment, in terms of lifetime, outperforms the uniform one. Indeed, in the greedy deployment, more sensors are deployed in virtual nodes with higher load while, in the uniform deployment, the same number of sensors are deployed in all virtual nodes, no matter their relative position to the sink.

When overhearing is not taken into account (Fig. 4a and 4b), we observe a significant increase in lifetime (more than 20 years) compared to a network with an overhearing ratio of 0.1 (about 3.5 years). This means that if we are able to use a perfect activity scheduling which reduces the overhearing, the network lifetime can increase significantly. Of course, this perfect scheduling would most likely require some supplementary control traffic which is not considered in our study.

In Fig. 4, the lifetime increases with the number of sensors. Increasing the total number of sensors in the network means to increase the number of sensors deployed per virtual node, both for the greedy and the uniform deployment. A direct consequence of increasing the number of sensors per virtual node is the reduction of the average number of transmissions per sensor, and thus prolonging the network lifetime. When the value of $p$ increases, the lifetime increases too. Indeed, since we assume a shortest-path routing, a high value of $p$ means a high probability for sensors in virtual node $B_{i}$ to relay traffic from $B_{i-2}$. Thus, by increasing the value of $p$, the amount of traffic relayed by $B_{i}$ from $B_{i-1}$ is reduced (See Eq. 1).

When $\alpha=0$, the average number of operations per sensor per time unit tends to 0 (see Eq. 15) when the number of sensors deployed in the virtual node is very large. That is why, in Fig. $4 a-4 b$, the lifetime is a strictly increasing linear function of the number of sensors. On the other hand, when overhearing is taken into account $(\alpha>0)$, the number of operations per node per time unit in the virtual node $B_{i}$ when the number of sensors is very large tends to $\alpha \cdot\left(2 \cdot T_{i}-\lambda+p \cdot T_{i-1}+T_{i+1}+p \cdot T_{i+2}\right)$. Thus, when this limit is reached, it is useless to deploy more sensors. That is why, in Fig. 4c-4d, we observe a threshold which is asymptotically reached starting from a given number of sensors. We can also notice in this figure that the gap between the lifetime of the greedy and the uniform deployment is reduced when more sensors are deployed. Indeed, the greedy deployment rapidly reaches the maximum lifetime compared to the uniform deployment.

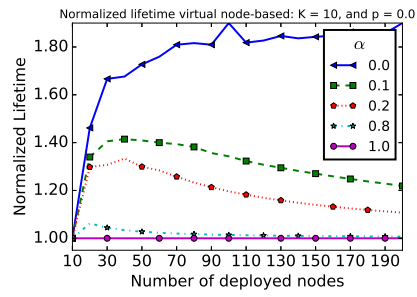

(a) $p=0.0$

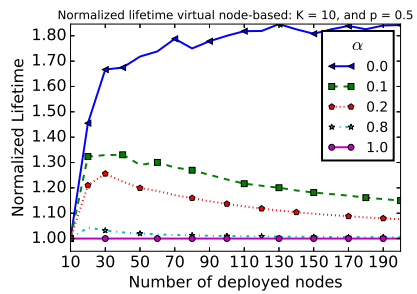

(b) $p=0.5$
FIGURE 5: Normalized lifetime, impact of the number of sensors

2) The lifetime gain of the greedy deployment: To illustrate how much the network lifetime could be extended when using the greedy Algorithm 1 rather than a simple uniform deployment, we normalize the lifetime of the greedy deployment by that of the uniform one. Thus, we use as metric the ratio $r$ expressed by Eq. 20 .

$$
r=\frac{L T^{(\text {greedy) }}}{L T^{\text {uniform })}}
$$


Fig. 5 shows, for different values of $\alpha$, the normalized lifetime of the network as a function of the number of sensors when the number of virtual nodes $(K)$ is equal to 10 . We consider the cases $p=0$ and $p=0.5$ in Fig 5a and 5b, respectively.

The normalized lifetime decreases when $\alpha$ increases. When there is no overhearing $(\alpha=0)$, the normalized lifetime increases continuously and reaches its maximum value around $N=90$ with an improvement of the lifetime by up to $80 \%$ compared to a uniform deployment. When there is no scheduling $(\alpha=1)$, i.e. all sensors always keep their radio on, the solution obtained with the greedy algorithm is equivalent to that given by a uniform deployment. This observation is consistent with our model and hypothesis, since Eq. 6, which expresses the average number of operations per node per time unit in a virtual node, shows that, when $\alpha=1, \bar{O}_{i}$ is independent of the number of sensors deployed in the virtual node $B_{i}$.

When $0<\alpha<1$, we observe, in Fig. 5, a considerable decrease of the performance compared to the case $\alpha=0$. Nevertheless, the lifetime of the uniform deployment can be improved by up to $40 \%$ (Fig. $5 \mathrm{a}, p=0$ ) or $30 \%$ (Fig. 5b, $p=0.5$ ), depending on the overhearing ratio. As expected, increasing the overhearing ratio $(\alpha)$ also increases the number of receptions in the network, and then reduces the lifetime of the network. We highlight that, from a given number of sensors, the performance of the greedy deployment compared to the uniform deployment starts decreasing. Indeed, as presented in the Fig. 4, from a given number of sensors, our greedy deployment reaches its maximum lifetime while the lifetime of the uniform deployment still increases, and thus, the gap between the greedy and the uniform deployment is reduced.

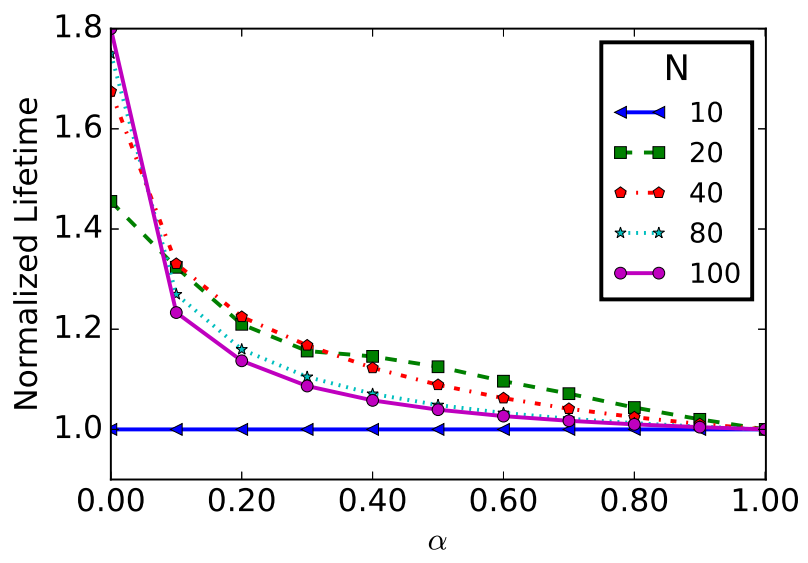

FIGURE 6: Impact of $\alpha: K=10$ and $p=0.5$

Fig. 6 presents the normalized network lifetime as a function of $\alpha$ and the number of deployed sensors. When the overhearing phenomenon is taken into account (i.e. $\alpha>0$ ), the gain (compared a deployment with $\alpha=0$ ) is reduced. Thus, when designing a deployment strategy or when evaluating the performance of communication protocols, it is important to take into account all messages that can be received by sensors. For values of $\alpha$ close to 1 , the greedy deployment is equivalent (or close) to a uniform deployment in terms of network lifetime.

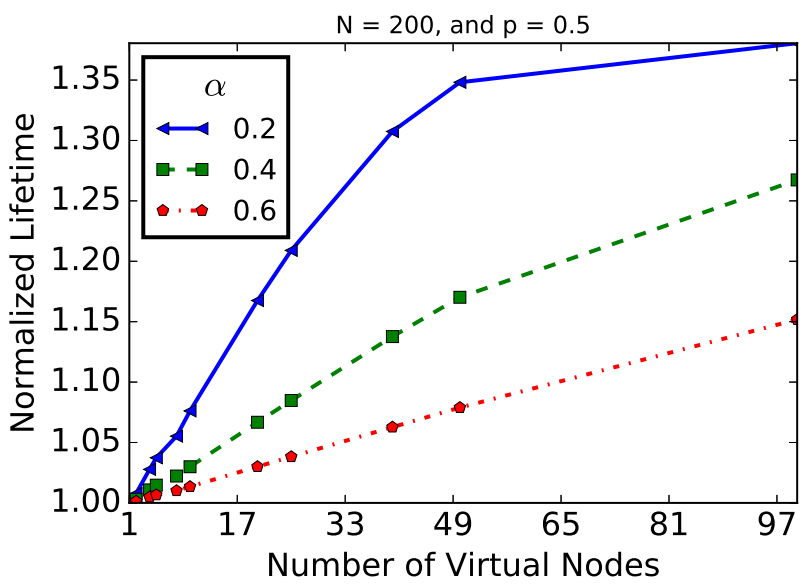

FIGURE 7: Impact of the network length $(K)$ for a network of $N=200$ sensors

3) Impact of the network length: Fig. 7 shows the impact of the network length, in terms of the number of virtual nodes. For a given number of sensors $(N=200)$, this figure shows the impact of the network length $(K)$ and the overhearing ratio $(\alpha)$ on the deployment proposed in this paper. Results presented in this figure show that the gap between the greedy deployment and the uniform one increases with $K$. Indeed, by increasing the $K$ value, the overall network traffic in the network increases too, and particularly in virtual nodes close to the sink. However, considering a uniform deployment, increasing the network length while keeping a constant number of sensors (as it is the case here) reduces the number of sensors to form each virtual node. A direct consequence is a negative impact on the network lifetime of the uniform deployment. It is not the case for the greedy deployment, since this algorithm deploys sensors in virtual nodes taking into account network traffic in each area.

\section{Residual energy}

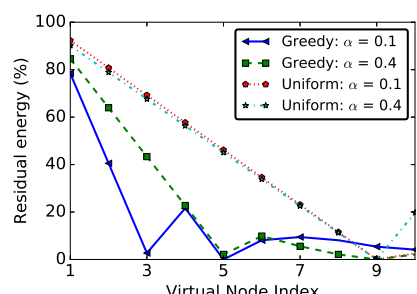

(a) $p=0.0$

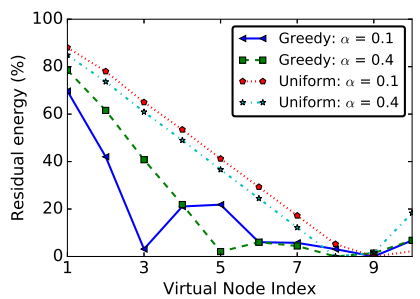

(b) $p=0.5$
FIGURE 8: Residual energy per sensor as function of the virtual node : $K=10, N=30$.

In this paper, we define the network lifetime as the time until the first sensor exhausts its energy. Considering a LWSN organized in $K=10$ virtual nodes, we present in this section the residual energy of sensors per virtual nodes at the end of the network functioning. In Fig. 8, results are given for $N=30, p=0.0$ (Fig. $8 \mathrm{a}$ ), $p=0.5$ (Fig. 8b) and for different values of $\alpha$. An important observation concerning the results presented in this figure is the highest residual energy of sensors for the uniform deployment. While the same number of sensors are deployed in each virtual nodes 
with the uniform deployment, our greedy deployment deploys more sensors in the area close to the sink. Thus, for virtual nodes far from the sink, the traffic per sensor is lower for the uniform deployment since it deployed more sensors compared to the greedy deployment proposed in this paper. This results in lower energy consumption for these sensors in the case of the uniform deployment compared to the greedy one. In many WSN, it is not possible to replace the sensor battery. In this case, when the network stops functioning, all the sensors are replaced. But, if only sensors with less energy where to be replaced, these results show that the battery of many sensors will be replaced for the greedy deployment than that for the uniform case. But, given the low-cost of sensors and the lifetime gain (see Fig. 5) of the greedy deployment compared to the uniform one, this is not a problem.

\section{E. Impact of the sink position}

So far, we assumed that the sink is deployed at position $K+1$. In this section, we evaluate the impact of the sink position on the network lifetime considering the uniform deployment as well as the greedy one proposed in this paper. We vary the sink position from 0 to $K+1$.

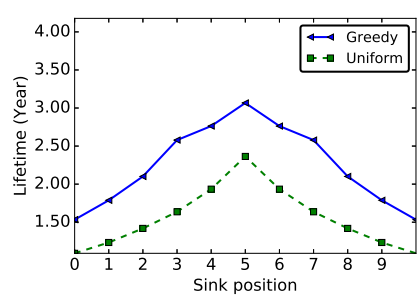

(a) $p=0.0$

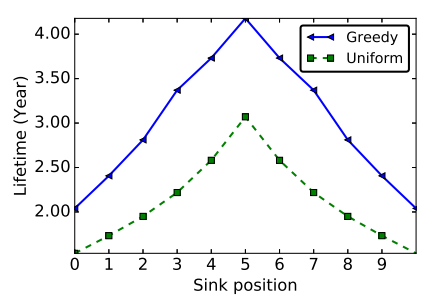

(b) $p=0.5$
FIGURE 9: Lifetime of the uniform and greedy deployments as function of the sink position : $K=10, \alpha=0.1, N=30$

Figure 9 shows the lifetime of the network considering the uniform and greedy deployments as function of the sink position. We consider a network formed of 10 virtual nodes, 30 sensors and $\alpha=0.1$. Results are shown for $p=0$ (Fig. 9a) and $p=0.5$ (Fig. 9b). From the network traffic point a view, the sink deployed at position 0 or $K+1$ corresponds to the configuration considered in the previous section and gives the same network lifetime. But, by deploying the sink at a different position than the network edges, the among of traffic forwarded by the closest neighbors of the sink is reduced. And as shown in Fig. 9, the optimal position is the middle of the network.

\section{COMPARISON TO RELATED WORK}

In this section, we compare the deployment strategy proposed in this paper to the one proposed in [8] assuming that the sink is deployed in the virtual node $K+1$. In the following, the solution proposed in this paper will be denoted as virtual node-based and the one proposed in [8] as distance-based. Since these two approaches are quite different, it is not easy to provide a fair comparison. In the following section, we start by describing the solution proposed by [8].

\section{A. Distance-based deployment}

In [8], the deployment is formulated as a Mixed-Integer Linear Programming (MILP) problem. In their formulation, the authors denote by $d_{i}$ the distance between sensors $S_{i}$ and $S_{i+1}$, and $R_{j}$ the communication range when the transmission power $P_{j}$ is used. For each sensor $S_{i}, m$ binary variables $\left(x_{i 1}, x_{i 2}, \cdots, x_{i j}, \cdots, x_{i m}\right)$ are defined to denote the assignment of power level for $S_{i}$. Thus :

$$
\begin{gathered}
\sum_{j=1}^{m} x_{i j}=1 \\
d_{i}=\sum_{j=1}^{m} x_{i j} \cdot R_{j}
\end{gathered}
$$

The authors assume that $S_{i}$ relays all the messages from $S_{i-1}$. If $\mu$ is the number of messages generated by a sensor per time unit, the number of operations (transmissions, since in [8] receptions are not taken into account) per sensor per time unit is defined by Eq. 23.

$$
\bar{O}_{i}^{(r)}=\mu \cdot i
$$

If we consider receptions and the overhearing phenomenon, as in this paper, the number of operations per sensor per time unit is now equal to :

$$
\begin{aligned}
\bar{O}_{i}^{(r)} & =\mu \cdot(i-1+i+\alpha \cdot(i+1)) \\
& =\mu \cdot(2 \cdot i+\alpha \cdot(i+1)-1)
\end{aligned}
$$

The energy consumed by $S_{i}$ per time slot is then equal to :

$$
E_{i}=\bar{O}_{i}^{(r)} \cdot \tau \cdot\left(\sum_{j=1}^{m} x_{i j} \cdot P_{j}\right)
$$

The object in [8] is to :

$$
\operatorname{minimize} \max \left\{E_{i} \mid i=1, \cdots, N\right\}
$$

subject to the following constraint :

$$
\sum_{i=1}^{N} x_{i j} \cdot d_{i} \geq L
$$

where $L$ is the initial network length, a parameter defined in [8]. We use the CPLEX software package to find the optimal solution to this problem. It is important to note here that, in the solution provided by the solver, the total area covered by sensors is larger than $L$ (see Eq. 27). In the following, we denote by $L_{\text {simulated }}$ the (final) length of the network provided by the solver.

In our work, we form a network of virtual nodes and we propose a greedy algorithm which calculates the number of sensors to deploy in each virtual node. The MILP problem proposed by [8] and described in this section (Eq. 26-27) considers a flat network and expresses the deployment results in terms of distance between consecutive nodes. 
TABLE III: Virtual node-based and distance-based comparison parameters

\begin{tabular}{|l|l|l|}
\hline Parameter & Distance-based & Virtual node-based \\
\hline $\begin{array}{l}\text { Communication } \\
\text { range }\end{array}$ & $R_{\min }$ to $R_{\max }$ & $R_{\max }$ \\
\hline $\begin{array}{l}\text { Number of virtual } \\
\text { nodes }\end{array}$ & - & $K=\left\lceil\frac{L_{\text {simulate }}}{R_{\max }}\right\rceil$ \\
\hline $\begin{array}{l}\text { Messages } \\
\text { generation rate }\end{array}$ & $\mu$ per node & $\begin{array}{l}\mu \cdot N \cdot \frac{R_{\max }}{L_{\text {simuled }}} \\
\text { per virtual node }\end{array}$ \\
\hline
\end{tabular}

\section{B. Comparison framework}

To provide a fair comparison, we use the same parameter values as the ones used in [8] to solve the problem described by Eq. 26 and 27. In our virtual node-based approach, without loss of generality, we assume as the average transmission power $P$, the maximum power level used in the distance-based approach. Thus, the area covered by a virtual node is of length $R_{\max }$, the transmission range when the maximum transmission power is used. Since [8] assumes that $S_{i}$ relays all traffic from $S_{i-1}$, we set $p=0$, i.e communications are not allowed between virtual nodes $B_{i}$ and $B_{i+2}$.

In the virtual node-based and the distance-based approaches, traffic models are different. In our evaluation, we guarantee the same average messages generation per sensor in both approaches. If, for the distance-based approach, $\mu$ is the average number of messages generated per sensor per time slot, the total number of messages generated in the network of length $L_{\text {simulate }}$ per time slot is $\mu \cdot N$, where $N$ is the total number of sensors. Thus, in our virtual node-based approach, we assume that $\lambda$, the average number of messages generated per virtual node per time slot is equal to $\mu \cdot N \cdot \frac{R_{\max }}{L_{\text {simulated }}}$. Comparison parameters are summarized in Table III.

\section{Comparison results}

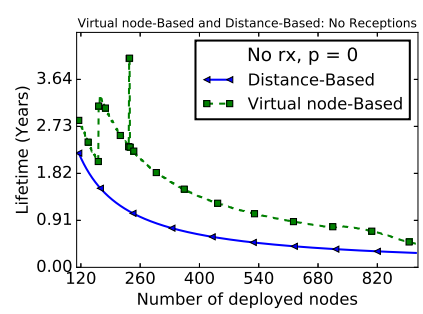

(a) No Receptions

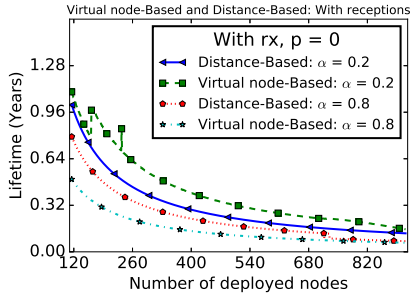

(b) With Receptions
FIGURE 10: Network lifetime of Distance-Based and Virtual node-Based deployment : $L=5 \mathrm{~km}$

Fig. 10 presents the lifetime of the network for distancebased and virtual node-based deployments. In Fig. 10a, only transmissions are considered, while Fig. 10b also takes into account receptions with and overhearing ratio of 0.2 and 0.8 . In the evaluations, we assume that $\Delta=1$ minute and $\mu=1 / \Delta$. As it might be expected, considering messages reception (Fig. $10 \mathrm{~b}$ ), the lifetime is divided by more than two compared to the model in which only transmissions are taken into account (Fig. 10a). When receptions are not considered (Fig 10a), the virtual node-based approach gives better performance compared to the distance-based one. This advantage of the virtual node-based approach is also noticed for low and medium overhearing ( $\alpha=$ 0.2 in Fig. 10b). In the virtual node-based approach, all sensors use the maximum power level. Thus, sensors are more exposed to the overhearing phenomenon. That is why, in Fig 10b, the distance-based approach gives better performance when $\alpha=$ 0.8 .

When the number of sensors in the network increases, the lifetime of the two approaches decreases. Indeed, deploying more sensors in the network has an impact on the two approaches. For the distance-based solution, this increases the traffic in the network (since each node generates messages) and the network length. When the network length increases, the number of virtual nodes increases too. Moreover, increasing the sensors density allows more sensors in the distance-based approach to use lower power level. That is why we observe a reduction of the gap between the two approaches in Fig. 10.

In Fig. 10, we observe a significant increase of the performance of our virtual node-based approach at some particular values of $N$. This is an artifact of the discrete power level model used in [8], where 6 power levels are possible. By increasing the number of sensors, smaller power levels are used with the distance-based approach. Thus, when a sufficient number of sensors is deployed, all sensors use the next lowest power level [8]. At these points, the resulting network length $L_{\text {simulated }}$ is almost equal to $L$, which reduces the number of virtual nodes and favors our solution.

\section{INVESTIGATING THE CASE OF HETEROGENEOUS SENSORS}

In the previous sections, we assumed we have a large number of homogeneous sensors. We divided the network into virtual nodes and then we proposed a greedy algorithm to compute the number of sensors to deploy in each virtual node in order to extend the network lifetime. In this section, the network is still divided virtual nodes, but only one sensor is deployed into each virtual node. We assume that sensors are heterogeneous in terms of battery capacity, and our goal is to find the battery capacity to assign to a given virtual node, taking into account its traffic, in order to extend the network lifetime. We also assume in this section that the sink is deployed in the virtual node $K+1$.

\section{A. Problem formulation}

We assume that we have an energy budget $E_{n e t}$, and our goal is to distribute this energy to all sensors, in order to maximize the network lifetime. We assume in this section that one sensor is deployed in each virtual node (i.e. $K=N$ ). If we replace $n_{i}=1$ in Eq. 15 , we will have a new model of the average number of operations per sensor per time unit, defined by the Eq. 28 :

$$
\bar{O}_{i}=\left(2 \cdot T_{i}-\lambda\right)+\alpha \cdot\left[p \cdot T_{i-1}+T_{i+1}+p \cdot T_{i+2}\right]
$$

We denote by $\xi_{i}$ the energy assigned to virtual node $B_{i}$. Since $E_{i}$ (Eq. 19) is the energy consumption rate of the virtual node $B_{i}$, the lifetime of this virtual node, $L T_{i}^{(\text {energy })}$, is defined by Eq. 29 :

$$
L T_{i}^{(\text {energy })}=\frac{\xi_{i}}{E_{i}}
$$


If we define the network lifetime $(L T)$ as the time until the first virtual node exhausts its energy, it will be defined by Eq. 30 :

$$
L T^{(\text {energy })}=\min \left\{L T_{i}^{(\text {energy })} \mid i=1, \cdots, K\right\}
$$

Our goal is to find $\xi_{i, 1 \leq i \leq K}$ which maximizes $L T^{(\text {energy })}$. Formally, the problem can be presented as :

$$
\text { maximize } L T^{(\text {energy })}
$$

subject to the following constraints :

$$
\begin{gathered}
\sum_{i=1}^{i=K} \xi_{i}=E_{n e t} \\
\xi_{i}>0
\end{gathered}
$$

Eq. 31-33 define a linear optimization problem. We find the optimal solution to this problem using the CPLEX software package.

Intuitively, the energy budget $E_{n e t}$ could be distributed among virtual nodes proportionally to their traffic. Thus, alternatively, we propose Eq. 34 to model the energy assigned to virtual node $B_{i}$.

$$
\xi_{i}=\frac{\bar{O}_{i}}{\sum_{j=1}^{j=K} \bar{O}_{j}} \cdot E_{n e t}
$$

In Eq. 34, $\bar{O}_{i}$ is the average number of operations per sensor per time unit in the virtual node $B_{i}$. Fig. 11 presents the optimal solution (provided by CPLEX software) to the problem described by Eq. 31-33 and the solution given by Eq. 34. This figure shows that the two solutions are almost identical.

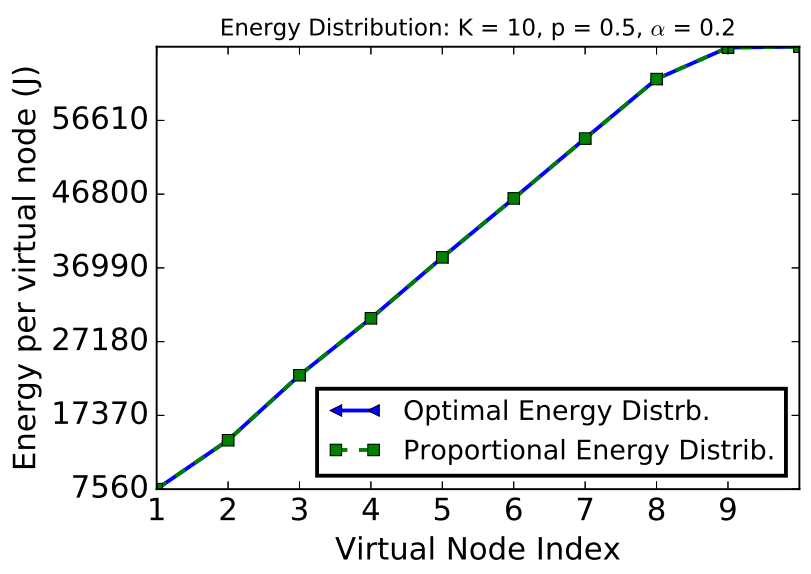

FIGURE 11: Optimal and proportional energy distribution per virtual node for an energy budget $E_{n e t}=408240 \mathrm{~J}$

Recall that $E_{i}=\bar{O}_{i} \cdot \tau \cdot P$. Thus, $L T_{i}^{(\text {energy })}$ is defined by Eq. 35 :

$$
\begin{aligned}
L T_{i}^{(\text {energy })} & =\frac{\xi_{i}}{E_{i}}=\frac{\bar{O}_{i}}{\sum_{j=1}^{j=K} \bar{O}_{j}} \cdot E_{n e t} \cdot \frac{1}{\bar{O}_{i} \cdot \tau \cdot P} \\
& =\frac{E_{n e t}}{\left(\sum_{j=1}^{j=K} \bar{O}_{j}\right) \cdot \tau \cdot P}
\end{aligned}
$$

Note in Eq. 35 that all sensors have the same lifetime and thus, will exhaust their battery at the same moment.

\section{B. Uniform vs proportional energy distribution}

For a uniform energy distribution, the same amount of energy is assigned to each virtual node. We define $\xi_{i}^{(u)}$, the energy assigned to each node, given by Eq. 36.

$$
\xi_{i}^{(u)}=\frac{E_{n e t}}{K}
$$

And $L T_{i}^{(\text {uniform })}$, the lifetime of the virtual node $B_{i}$ for a uniform energy distribution, is defined by Eq. 37 .

$$
L T_{i}^{(\text {uniform })}=\frac{\xi_{i}^{(u)}}{E_{i}}=\frac{E_{n e t}}{K} \cdot \frac{1}{\bar{O}_{i} \cdot \tau \cdot P}=\frac{E_{n e t}}{K \cdot \bar{O}_{i} \cdot \tau \cdot P}
$$

If the lifetime of the optimal energy distribution is normalized by the lifetime given by the uniform energy distribution, we obtain :

$$
\begin{aligned}
\frac{L T_{i}^{(\text {energy })}}{L T_{i}^{(\text {uniform })}} & =\frac{E_{n e t}}{\left(\sum_{j=1}^{j=K} \bar{O}_{j}\right) \cdot \tau \cdot P} \cdot \frac{K \cdot \bar{O}_{i} \cdot \tau \cdot P}{E_{n e t}} \\
& =\frac{\bar{O}_{i} \cdot K}{\sum_{j=1}^{j=K} \bar{O}_{j}}
\end{aligned}
$$

Thus, if different energy capacities can be assigned to sensors, the optimal capacity assignment (Eq. 34) can improve the lifetime of the virtual node $B_{i}$ by a factor of $\frac{\bar{O}_{i} \cdot K}{\sum_{j=1}^{j=K} \bar{O}_{j}}$ compared to a uniform energy distribution. If we denote by $\bar{O}_{\max }=\max \left\{\bar{O}_{i} \mid i=1, \cdots, K\right\}$, the maximum number of operations per sensor per time unit in the network, we obtain : $\bar{O}_{\max } \cdot K>\sum_{j=1}^{j=K} \bar{O}_{j}$ and $\frac{\bar{O}_{\max } \cdot K}{\sum_{j=1}^{j=K} \bar{O}_{j}}>1$.

\section{Deployments based on heterogeneous and homogeneous sensors}

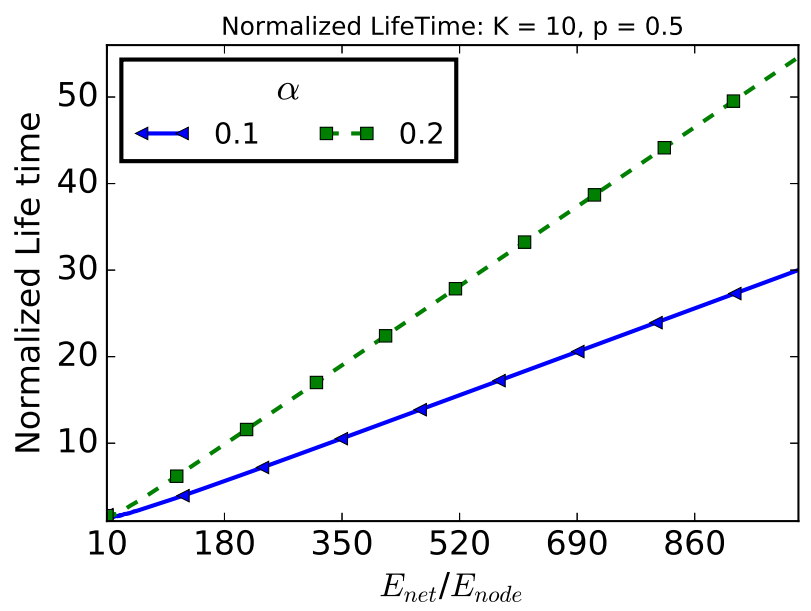

FIGURE 12: Energy distribution vs Virtual nodes-based : Impact of the energy budget when $K=10, p=0.5$.

Our goal here is to compare the deployment based on energy distribution solution proposed in this section to the one based on virtual nodes described by Algorithm 1. Recall that, given $N$ sensors, this greedy algorithm finds the number of sensors to deploy in each virtual node. To allow a fair comparison, we assume that the energy unit is equal to $E_{\text {node }}$, and an energy budget of $E_{\text {net }}$ can be discretized 
to have $N=\frac{E_{n e t}}{E_{\text {node }}}$ homogeneous sensors. Thus, given the energy budget, we can fairly compare the energy distribution deployment to the sensors distribution one by discretizing the energy budget and implementing the two solutions.

Fig. 12 presents $\frac{L T^{(\text {energy) }}}{L T^{(\text {greedy })}}$ as a function of $\frac{E_{n e t}}{E_{\text {node }}}$ (recall that $L T^{(\text {greedy) }}$ is the network lifetime obtained by Algorithm 1). This figure shows that the energy distribution solution outperforms the sensor distribution one and the gap increases with the ratio $\frac{E_{n e t}}{E_{\text {node }}}$. It is also important to note that, by deploying a single sensor in each virtual node, the routing problem is simplified and there is no load balancing problem of the traffic per virtual node as in the case of the solution proposed in the previous sections. However, it may not be economically or practically feasible for industrials to produce sensors with different batteries.

\section{CONCLUSION}

In this paper, we studied the problem of sensors deployment to maximize the lifetime of WSN with linear topology. To simplify the deployment, we divided the network into virtual nodes. By considering messages transmission and/or reception as energy operations consuming, we proposed an analytical model of the number of operations per virtual node. Unlike existing works, our model considers the message reception cost. It also takes into account messages received due to the overhearing phenomenon.

Considering homogeneous sensors in terms of battery capacity, we proposed a greedy algorithm to compute the number of sensors to deploy in each virtual node. Performance evaluation shows that, depending on the number of sensors, the simple virtual node-based deployment proposed can improve the network lifetime by up to $40 \%$, when compared to the uniform deployment. Moreover, it outperforms the distancebased approach when a scheduling algorithm which reduces the overhearing phenomenon is used.

Finally, we studied an alternative solution. We considered a network in which the batteries of sensors are nonuniform, i.e. the battery of a sensor is proportional to its traffic. Results show that, by properly selecting the battery to assign to each sensor, such an approach significantly improves the network lifetime.

Our model assume an average transmission power used by all sensors in a virtual node. However, existing radio modules provide many transmission power levels that can be configured by programming. In our future work, we plan to integrate this into our model by defining the output power level to be used by each sensor deployed in a virtual node.

\section{RÉFÉRENCES}

[1] I. Jawhar, N. Mohamed, D.P. Agrawal, "Linear Wireless Sensor Networks : Classification and Applications", Journal of Network and Computer Applications, 34(5) :1671-1682, Sep. 2011.

[2] F. Stajano, N. Hoult, I. Wassell, P. Bennett, C. Middleton, K. Soga, "Smart Bridges, Smart Tunnels : Transforming Wireless Sensor Networks from Research Prototypes into Robust Engineering Infrastructure", Ad Hoc Networks, 8(8) :872-888, Nov. 2010.

[3] W. Fisher, T. Camp, V. Krzhizhanovskaya, "Crack Detection in Earth Dam and Levee Passive Seismic Data Using Support Vector Machines", Proc. ICCS 2016, San Diego, CA, USA, Jun. 2016.
[4] R. Domga Komguem, R. Stanica, M. Tchuente, F. Valois, "WARIM : Wireless Sensor Networks Architecture for a Reliable Intersection Monitoring", Proc. IEEE ITSC 2014, Quingdao, China, Oct. 2014.

[5] Z. Sun, P. Wang, M. Vuran, M. Al-Rodhaan, A. Al-Dhelaan, I. Akyildiz, "BorderSense : Border Patrol through Advances Wireless Sensor Networks", Ad Hoc Networks, 9(3) :468-477, May 2011.

[6] M. Perillo, Z. Cheng and W. Heinzelman, "On the problem of unbalanced load distribution in wireless sensor networks," IEEE Global Telecommunications Conference Workshops, November 2004, Dallas, USA, pp. 74-79.

[7] M. Noori and M. Ardakani, "Characterizing the traffic distribution in linear wireless sensor networks", IEEE Communications Letters, vol. 12, no. 8, pp. 554-556, Aug. 2008.

[8] Y. Guo, F. Kong, D. Zhu, A. Å. Tosun, and Q. Deng (2010, April). "Sensor placement for lifetime maximization in monitoring oil pipelines." In Proceedings of the 1st ACM/IEEE International Conference on CyberPhysical Systems (pp. 61-68).

[9] S. Olariu and I. Stojmenovic. "Design guidelines for maximizing lifetime and avoiding energy holes in sensor networks with uniform distribution and uniform reporting“. In INFOCOM 2006, pages 1â12, April 2006

[10] C. Ok, H. Thadakamalla, U. Raghavan, S. Kumara, S. G. Kim, X. Zhang, and S. Bukkapatnam (2007, September). "Optimal transmission power in self-sustainable sensor networks for pipeline monitoring." In IEEE International Conference on Automation Science and Engineering (CASE), 2007, (pp. 591-596).

[11] Liu, Xin, and Prasant Mohapatra. "On the deployment of wireless data back-haul networks.” IEEE Transactions on Wireless Communications 6.4 (2007).

[12] K. Aberer and M. Hauswirth and A. Salehi. "A middleware for fast and flexible sensor network deployment." In Proceedings of the 32nd international conference on Very large data bases, September 2006.

[13] N. Raveendranathan and S. Galzarano and V. Loseu and R. Gravina and R. Giannantonio and M. Sgroi and R. Jafari and G. Fortino. "From modeling to implementation of virtual sensors in body sensor networks." IEEE Sensors Journal, 12(3), pp.583-593, 2012

[14] S. Madria and V. Kumar and R. Dalvi, "Sensor Cloud : A Cloud of Virtual Sensors.” In IEEE Software, vol. 31, no. 2, pp. 70-77, Mar.-Apr. 2014.

[15] T. S. Rappaport. "Wireless Communications : Principles and Practise.“ Prentice Hall, 1996.

[16] H. Yetgin, and K. T. K. Cheung, and M. El-Hajjar and L. H. Hanzo, "A Survey of Network Lifetime Maximization Techniques in Wireless Sensor Networks, “IEEE Communications Surveys \& Tutorials, vol. 19, no. 2, pp. 828-854, Second quarter 2017.

[17] P. Vicaire, T. He, Q. Cao, T. Yan, G. Zhou, L. Gu, L. Luo, R. Stoleru, J. A. Stankovic, and T. F. Abdelzaher. Achieving long-term surveillance in vigilnet. ACM Transaction Sensor Networks, 5(1) :1â39, 2009

[18] C. Sevgi and A. KoÃßyiÄit. "Optimal deployment in randomly deployed heterogeneous WSNs : A connected coverage approach", Journal of Network and Computer Applications, 46, pp.182-197, 2014.

[19] M.Z.A. Bhuiyan and G. Wang and J. Cao and J. Wu, "Deploying wireless sensor networks with fault-tolerance for structural health monitoring", IEEE Transactions on Computers, 64(2), pp.382-395, 2015

[20] F.J. Parrado-Garcia and J. Vales-Alonso and J.J. Alcaraz, "Optimal Planning of WSN Deployments for In SituLunar Surveys", IEEE Transactions on Aerospace and Electronic Systems, 53(4), pp.1866-1879, 2017.

[21] A. Boubrima and W. Bechkit and H. Rivano, "Optimal WSN deployment models for air pollution monitoring”, IEEE Transactions on Wireless Communications, 16(5), pp.2723-2735, 2017.

[22] N. Kulkarni and N.R. Prasad and R. Prasa, "A novel sensor node deployment using low discrepancy sequences for WSN", Wireless Personal Communications, 100 (2), pp.241-254, 2018.

[23] S. Potthuri and T. Shankar and A. Rajesh, âLifetime improvement in wireless sensor networks using hybrid differential evolution and simulated annealing (DESA)â. Ain Shams Engineering Journal, Elsevier, Volume 9, Issue 4,Pages 655-663, 2018

[24] K.R. Domga, and R. Stanica, and M. Tchuente, and F. Valois "Nodes Ranking in Wireless Sensor Network with Linear Topology“, IEEE WD'2017, Porto, Portugal, March, 2017 
[25] S. Plancoulaine, and A. Bachir, and D. Barthel, "WSN Node energy dissipation“, Technical repport, France Telecom R\&D, Internal Repport, July 2006

[26] A. M. Zahid and A.B.Kamalrulnizam and A. Muhammad and M.. Hafiz, "An overview of routing techniques for road and pipeline monitoring in linear sensor networks“, Wireless Networks, 2018

[27] W. Ye, and J. Heidemann, and D. Estrin, "Medium access control with coordinated adaptive sleeping for wireless sensor networks", IEEE/ACM Trans. Netw., vol 12, no. 3, pp. 493-506, June 2004.

[28] Y. Zhao, and J. Wu, and F. Li and S. Lu, "On maximizing the lifetime of wireless sensor networks using virtual backbone scheduling“, IEEE Trans. Parallel Distrib. Syst., vol. 23, no. 8, pp. 1528-1535, Aug. 2012.

[29] Y. Chen, and Q. Zhao, and V. Krishnamurthy and D. Djonin, "Transmission scheduling for optimizing sensor network lifetime : A stochastic shortest path approach“, IEEE Trans. Signal Process., vol. 55, no. 5, pp. 2294-2309, May 2007 